\title{
Lack of exon 10 in the murine tau gene results in mild sensorimotor defects with aging
}

\author{
Astrid Gumucio ${ }^{1}$, Lars Lannfelt ${ }^{1}$ and Lars NG Nilsson ${ }^{1,2^{*}}$
}

\begin{abstract}
Background: Complex species-specific, developmental- and tissue-dependent mechanisms regulate alternative splicing of tau, thereby diversifying tau protein synthesis. The functional role of alternative splicing of tau e.g. exon 10 has never been examined in vivo, although genetic studies suggest that it is important to neurodegenerative disease.

Results: Gene-targeting was used to delete exon 10 in murine tau on both alleles (E10-/-) to study its functional role. Moreover, mice devoid of exon 10 (E10+/-) on one allele were generated to investigate the effects of 1:1 balanced expression of $4 R-/ 3 R$-tau protein, since equal amounts of $4 R-/ 3 R$-tau protein are synthesized in human brain. Middle-aged E10-/- mice displayed sensorimotor disturbances in the rotarod when compared to age-matched E10+/- and wild-type mice, and their muscular grip strength was less than that of E10+/- mice. The performance of E10+/- mice and wild-type mice (E10+/+) was similar in sensorimotor tests. Cognitive abilities or anxiety-like behaviours did not depend on exon 10 in tau, and neither pathological inclusions nor gene-dependent morphological abnormalities were found.

Conclusion: Ablation of exon 10 in the murine tau gene alters alternative splicing and tau protein synthesis which results in mild sensorimotor phenotypes with aging. Presumably related microtubule-stabilizing genes rescue other functions.
\end{abstract}

Keywords: Alzheimer's disease, Tau, Knockout mice, Alternative splicing, Microtubule

\section{Background}

Tau belongs to the family of microtubule-associated proteins (MAPs), which bind to and/or interact with microtubule. It has been suggested that tau and other MAPs serve to promote assembly of microtubule to make them structurally stably, yet dynamic [1]. Disruption of the tau gene led to structural abnormalities of microtubule organization in small-calibre axons [2], and behavioural deficits with hyperactivity, impaired motor strength and coordination [3]. In contrast with the initial report [2], there were in vitro phenotypes with delayed development of neuronal polarity and formation of axons in cultured embryonal hippocampal neurons from tau knockout mice [4]. Functional redundancy, particularly between tau and map genes likely exists, since mapla protein expression was enhanced in tau knockout mice [2,4]. Indeed

\footnotetext{
* Correspondence: Lars.Nilsson@medisin.uio.no

'Department of Public Health and Caring Sciences, Rudbeck Laboratory, Uppsala University, SE-75185 Uppsala, Sweden

${ }^{2}$ Department of Pharmacology, Oslo University and Oslo University Hospital, Postboks 1057 Blindern, NO-0316 Oslo, Norway
}

tau/map1b double knockout mice displayed brain anomalies with severe defects on axon tract and neuronal layer formation, together with abnormal growth cone morphology and pronounced microtubule disorganization in primary neurons derived from these mice [5]. Tau protein synthesis and function is regulated by alternative splicing in a complex species-specific, developmental- and tissuedependent manner [6,7]. Depending upon the inclusion or exclusion of exon 10, tau mRNA isoforms with three (3R-tau mRNA) and four (4R-tau mRNA) microtubule binding domains are generated, and in total six tau mRNA isoforms are produced in the human brain. In the adult human brain, splicing is balanced with a 1:1 expression of $4 R$ - and 3R-tau mRNA $[8,9]$. This is in contrast to mouse brain in which only $3 R$-tau mRNA is generated at birth and only $4 R$-tau mRNA is synthesized at adulthood $[7,10,11]$. Thus equal amounts of $4 \mathrm{R}$ - and $3 \mathrm{R}$-tau protein is synthesized in adult human brain, while only $4 \mathrm{R}$-tau protein is produced in adult mouse brain. Interestingly, mutations in exon 10 or in adjacent regulatory sequences, can give rise to neurodegenerative disease with accumulation of

\section{() Biomed Central}


filamentous inclusions of tau in the human brain. Regulation of alternative splicing of tau is very complex and partly unknown. In order to investigate the functional role of exon 10 in murine tau we ablated the gene on both alleles, and thereby generated E10-/- mice which should in theory only synthesize 3R-tau protein. Moreover, the effect of a 1:1 balanced 3R-/4R-tau mRNA splicing of human brain was examined by generating mice in which exon 10 in tau was deleted on only one allele $(\mathrm{E} 10+/-)$. E10+/- mice should in theory synthesize equal amounts of 3R- and 4R-tau protein, as in the human brain (Additional file 1: Figure S1). The effects of fine-tuned regulation of alternative splicing being critical to maintain neuronal functions and viability could thereby be assessed [12].
Here we show that E10-/- but not E10+/- mice, display impaired sensorimotor abilities with aging.

\section{Results}

Generation of mice devoid of exon 10 in murine tau

Genomic regions (2.2 and $3.6 \mathrm{kbp}$ ) framing exon 10 in the murine tau gene were amplified with high-fidelity long-range PCR and subcloned into a pBluescript II $\mathrm{KS}$-vector that harboured a loxP-neomycin-loxP-cassette (Figure 1A). The gene targeting construct was checked by multiple restriction enzyme digests and sequencing of both introns and the intervening neomycin cassette. The gene-targeting vector was made linear with SspI and introduced into R1 embryonic stem (ES) cell with

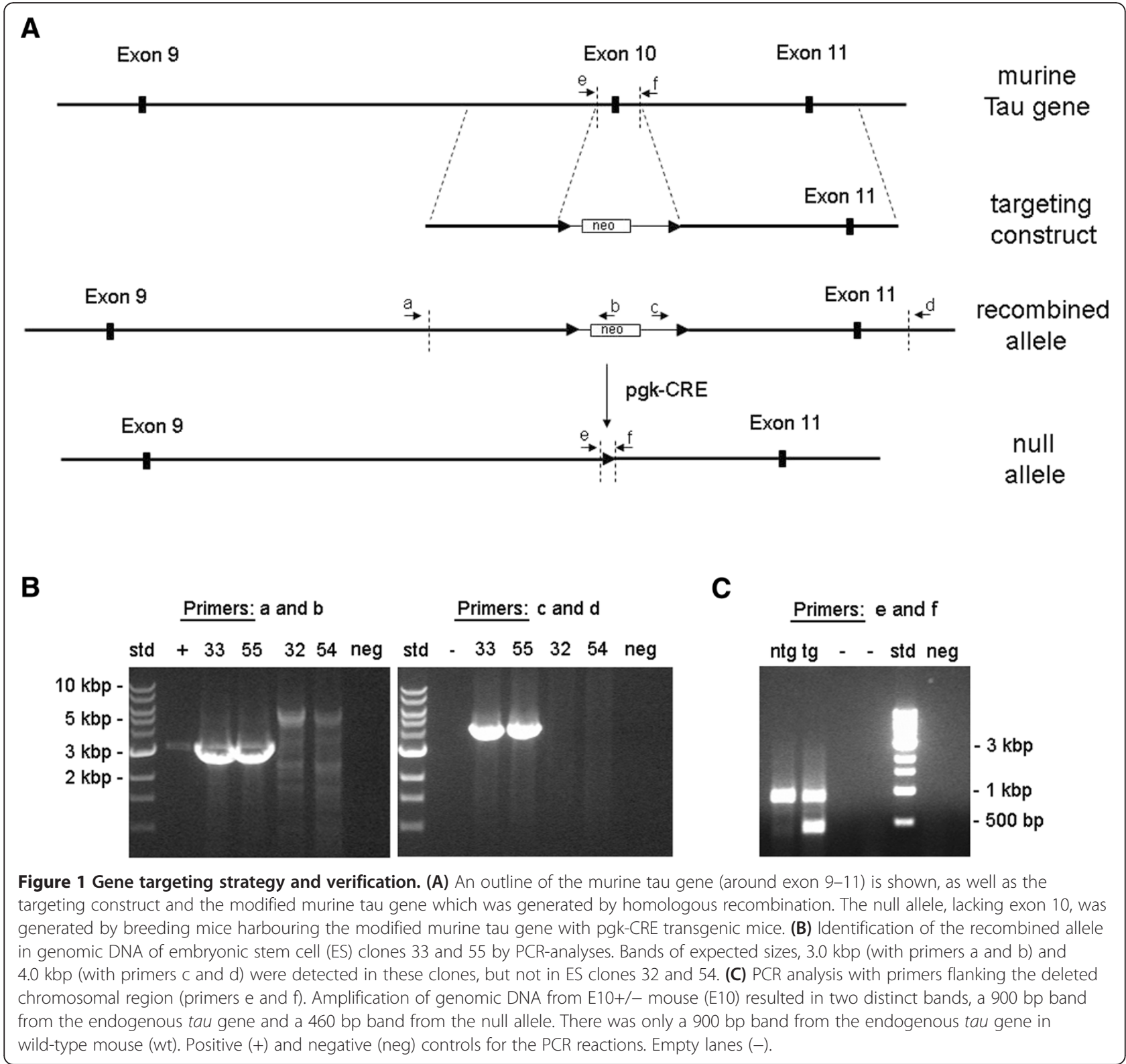


electroporation. ES cell clones were screened for homologous recombination and in a few clones, integration in the murine tau locus led to deletion of exon 10 and adjacent intron sequences ( $200 \mathrm{bp}$ on each side of exon 10). The clones were screened with PCR-reactions in which one primer was located outside the homologous sequences of the targeting construct. Those experiments resulted in $3.0 \mathrm{kbp}$ and $4.0 \mathrm{kbp}$ bands respectively (Figure 1B). One of the positive ES cell clones was microinjected into blastocysts and transplanted into pseudopregnant mice. Male chimeric mice were bred with $\mathrm{C} 57 \mathrm{Bl} / 6 \mathrm{~J}$ and their offspring were examined for evidence of germ line transmission. These were further bred with transgenic mice expressing phosphoglycerate kinase (pgk)-CRE recombinase to delete the neo cassette. PCR with genomic DNA and primers flanking exon 10 provided evidence of an endogenous tau allele (900 bp) and a null allele (460 bp) in E10+/ - mice, while only a single band was present in wild-type siblings (E10+/+; Figure 1C).

\section{Lack of exon 10 in tau results in 3R-tau protein synthesis in adult mouse brain}

Next we investigated E10+/- mice for expected deviations in tau mRNA expression and protein synthesis. RT-PCR analyses were performed with primers located within exon 9 and exon 11 to generate PCR products of $305 \mathrm{bp}$ (4R-tau mRNA) and 212 bp (3R-tau mRNA) respectively. As expected, a 1-day-old wild-type mouse (wt; P1) expressed only 3R-tau mRNA, while a 9-days-old wild-type mouse (P9) generated equal amounts of $3 R$ - and $4 R$-tau mRNA due to alternative splicing (Figure 2A). An 18days-old wild-type mouse (P18) produced only $4 R$-tau mRNA. In contrast, a 2-months-old E10+/- mouse (E10) generated $3 R$ - and $4 R$-tau mRNA in essentially equal proportions, while only $4 R$-tau mRNA was produced in an age-matched wild-type mouse (Figure 2A). Protein synthesis was examined with RD3 antibody, which is selective for 3R-tau, by western blot and immunohistochemistry. On western blot, all three isoforms of 3Rtau were found in E10+/- (E10) mouse brain, but the isoform devoid of exon 2 and 3 was the predominant (Figure 2B and Additional file 1: Figure S1). Tau-1, an antibody which recognizes both 3R- and 4R-tau, generated extra protein bands in E10+/- (E10) mice as compared to wild-type (wt) mice (Figure $2 \mathrm{C}$ ). The results were consistent with 3R-tau protein synthesis in E10+/- (E10) mouse brain. Immunohistochemistry with RD3 antibody resulted in strong staining in the stratum radiatum in CA1-CA3 areas of the hippocampus and faint staining in the soma of pyramidal neurons in adult E10+/- mice (Figure 2D), but not in wild-type mice who did not express 3R-tau protein (Figure 2E). In contrast, the non-selective Tau-1 antibody showed a staining pattern consistent with tau being mainly confined to the axonal compartment [13] in both adult E10+/- (Figure 2F) and in wild-type mice (Figure 2G). There was no immunostaining in the absence of a primary antibody (Figure $2 \mathrm{H}-\mathrm{I}$ ).

\section{Absence of exon10 in tau results in age-dependent sensorimotor dysfunctions}

Subsequently, we aimed to investigate the physiological and functional role of exon 10 in tau. In previous reports, tau knockout mice developed sensorimotor dysfunctions due to loss of tau protein synthesis [3]. Cerebellum, which is implicated in balance and motor learning, develops at postnatal stages when $4 \mathrm{R}$-tau is normally produced. We therefore examined whether partial or complete absence of exon 10 in tau led to sensorimotor anomalies in adult (5-6 months) and middle-aged (13-17 months) gene-manipulated mice. Rotarod and grip strength meter were used to assess sensorimotor dysfunctions. Genotype-dependent motor coordination dysfunction was not detected among adult mice (Figure 3A, $\mathrm{F}(2,50)=0.27$; $\mathrm{p}=0.76$ ), while differences in rotarod performance were observed among middle-aged mice $(\mathrm{F}(2,46)=4.34 ; \mathrm{p}<0.05)$. Post-hoc analyses showed that E10-/- mice were impaired compared to E10+/+ $(\mathrm{p}<0.05)$ and E10+/- $(\mathrm{p}<0.01$, Figure $3 \mathrm{~B})$. Similar results were found when the highest speed at which the animals remained on the rotarod was used as outcome measure (Additional file 1: Figure S2, $\mathrm{F}(2.46)=5.05 ; \mathrm{p}<0.05)$. Post-hoc analyses revealed that the muscular strength of adult E10+/- mice was superior to that of $\mathrm{E} 10+/+$ mice (Figure $3 \mathrm{C}$; $\mathrm{p}<0.05$ ). In contrast, middle-aged E10-/ - mice were weaker than $\mathrm{E} 10+/-(\mathrm{p}<0.05)$, but not $\mathrm{E} 10+/+$ mice $(\mathrm{p}=0.10$; $\mathrm{F}(2,45)=2.43$; Figure 3D).

E10-/- mice are less active than E10+/- mice in IntelliCage The effect of partial and complete deletion of tau exon 10 on general behaviour and cognitive function was assessed in IntelliCages, a new non-invasive system for behavioural phenotyping $[14,15]$. Since the behaviours of $\mathrm{E} 10+/-$ and wild-type mice $(\mathrm{E} 10+/+)$ in sensorimotor tests were essentially equal, we chose to only examine two experimental groups, 9-10 months-old E10+/- and E10-/- mice. Moreover we wanted to increase group size and statistical power. First, tendency to explore and habituate to novel environment was examined. During the first 2 hours of the study, the LEDs were turned on in two of the four corners. The E10-/- mice $(61.2 \pm 4.2 \%, \mathrm{n}=10)$ spent more time in the illuminated corners than $\mathrm{E} 10+/-$ mice $(46.6 \pm 4.8 \%, \mathrm{n}=9 ; \mathrm{t}(17)=-2.31$, $\mathrm{p}<0.05)$. During the first 24 hours, E10+/- mice (64 \pm 17 , $\mathrm{n}=10$ ) tended to perform more visits to the corners than E10-/- mice $(28 \pm 10, \mathrm{n}=12$; Mann Whitney $\mathrm{U}=31, \mathrm{p}=0.06$ ). As previously observed, there was a marked diurnal activity pattern, i.e. more activity 


\section{overexposure}
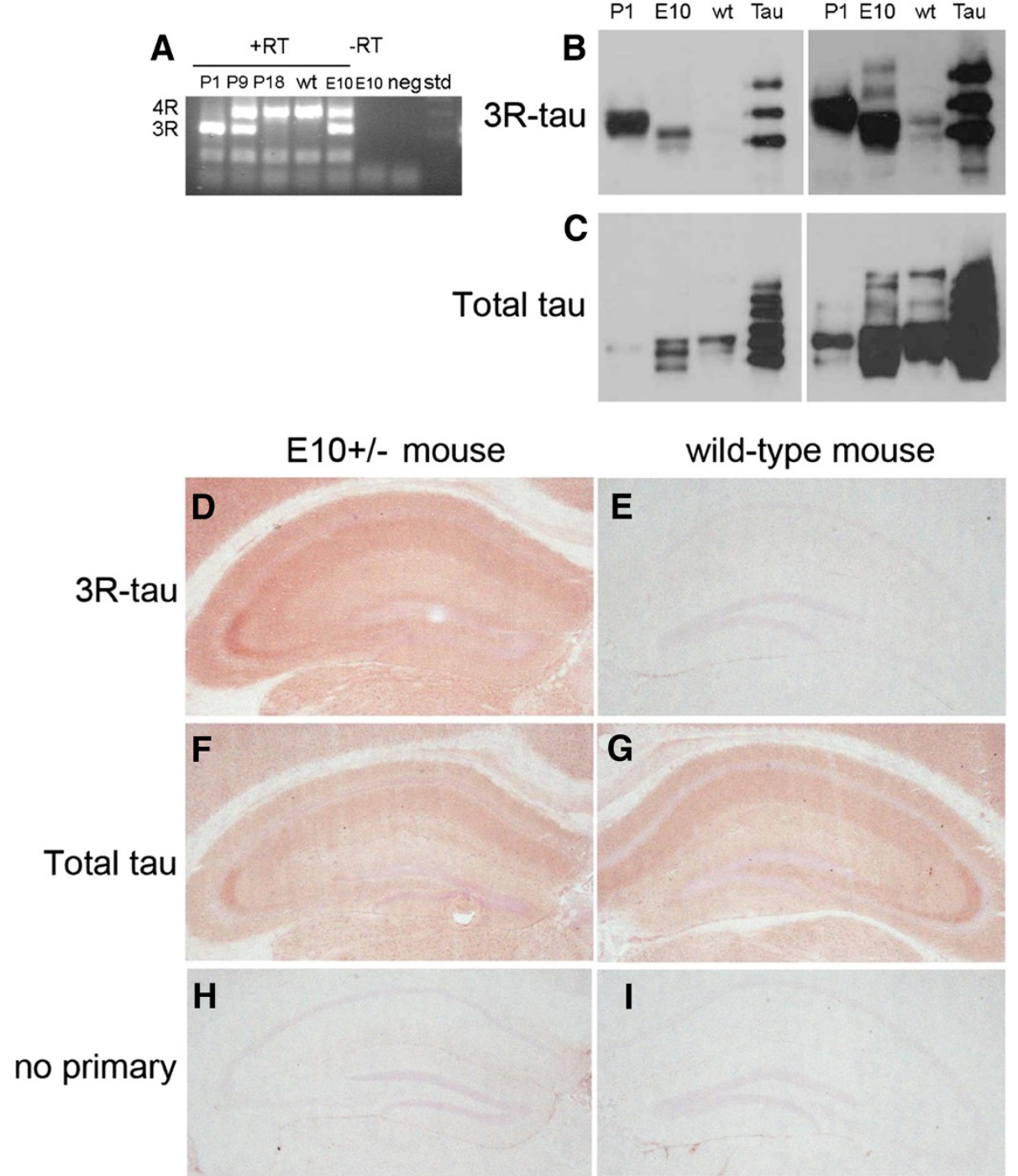

Figure 2 Tau mRNA and protein expression in E10+/- and wild-type mice. (A) Only 3R-tau mRNA (212 bp) was expressed at birth (P1) in wild-type mice (wt). At (P9), 3R-tau and 4R-tau mRNA (305 bp) was expressed, while at (P18) and in 2-month-old wt-mice, exon 10 was included and $4 R$ tau mRNA was expressed. In contrast, 2-month-old E10+/- mice (E10) produced 3R-tau and 4R-tau mRNA in equal proportions. Signals depended on mRNA and reverse transcriptase in the cDNA synthesis reaction (-RT, E10). (B) Left; Western blot with a selective antibody for 3R tau resulted in a $38 \mathrm{kDa}$ band in 2 months-old E10+/- mouse brain (E10). Right; Two additional bands were seen when films were overexposed. These bands likely represented tau-isoforms with $\mathrm{N}$-terminal domains exon 2 and 3 . In an age-matched wt-mouse there was only a small amount of 3R-tau protein. (C) Left; An antibody (Tau-1) that recognizes 3R-tau as well as 4R-tau generated two protein bands at 38 and $39 \mathrm{kDa}$ in brains of 2-month-old E10+/- mice (E10). Right; Overexposure results in two additional double bands likely representing isoforms of tau with the N-terminal domains exon 2 and 3 of tau protein. Only a small amount of 3R tau protein was found in age-matched wild-type littermates (wt). Recombinant human tau protein representing all tau isoforms and a newborn wild-type mouse brain (P1) was included as a positive control. (D) Immunostaining with selective antibody 3RD showed that 3R-tau was mainly localized in axons of E10+/- mice, (E) but not present in an age-matched wild-type mice. (F-G) The non-selective antibody Tau-1, which recognizes both 3R-tau and 4R-tau, stained hippocampal layers which are rich in axons in both E10+/- (F) and wild-type mice (G). (H-I) No immunostaining in E10+/- (H) or wild-type mice in the absence of primary antibody (I).

during the dark phases compared to light phases. There was a significant time-dependent decrease in the number of corner visits $(\mathrm{F}(13,260)=7.0, \mathrm{p}<0.001)$ indicating that E10+/- and E10-/- habituated to the new environment (Figure 4A). Interestingly, during the habituation, E10+/- mice were significantly more active than E10-/- mice $(\mathrm{F}(1,20)=8.87, \mathrm{p}<0.01$; Figure $4 \mathrm{~A})$. The amount of time spent drinking, measured as lick duration, increased over days for both groups of mice $(\mathrm{F}(13,260)=6.74, \mathrm{p}<0.001$, data not 


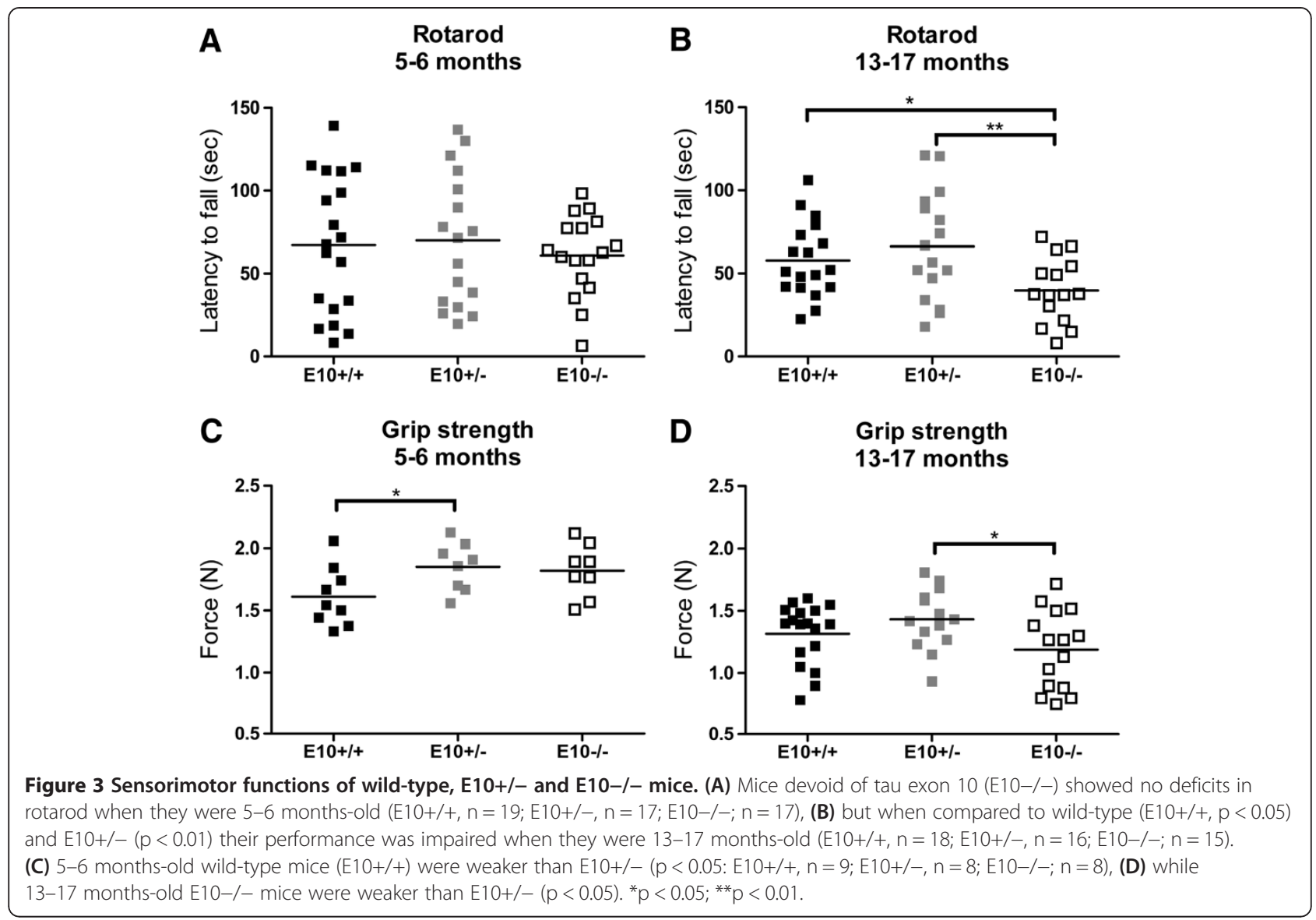

shown), but the length of lick duration did not differ between genotypes. Despite a habituation effect, three E10-/- mice performed few corner visits and drank little, and therefore had to be excluded. They were replaced by four new E10-/- mice, which were allowed to habituate to the IntelliCages for additional 7 days. The inserted mice were initially very active, with high numbers of corner visits. In the following days, the number of corner visits drastically decreased. Their activity was not significantly different from the rest of the mice during the last three days of the habituation.

\section{Place learning or associative learning do not depend on the presence of exon 10 in tau}

The IntelliCages are equipped with four corners, each with two doors leading to their respective water bottle. In the Place learning protocol, an error was defined as a nosepoke on any of the seven doors where sucrose water (reward) was not available, i.e. the closed side of each corner (=four doors) and the accessible side in the three corners where only water was available (=three doors). Any visit to a door where reward was not provided was defined as an erroneous behaviour. The percentage of errors made by E10-/- mice decreased from the first day of testing to the last day (from $84.2 \pm$ $4.6 \%$ to $63.6 \pm 8.9 \%, \mathrm{p}=0.06$; chance level is $87.5 \%$ ). Likewise, the percentage of errors decreased for E10+/- mice from $95.3 \pm 2.3 \%$ to $79.8 \pm 6.0 \%$ ( $<<0.05)$. However, spatial learning ability did not depend on genotype (Figure $4 \mathrm{~B}$ ). During reversal learning, when reward was provided behind the diagonally opposite door, E10-/- mice made more errors on first $84.2 \pm 5.5 \%$ than on the last day of testing $59.7 \pm 8.7 \%(\mathrm{p}<0.05$; chance level is $87.5 \%)$, but this was not observed for E10+/- mice $(82.8 \pm 5.7 \%$ to $79.7 \pm 6.2 \%, p=0.72$; Figure 4C). Again, reversal learning did not depend on genotype. When mice again could access water behind all eight doors (extinction of Place learning, 12 hours), E10+/- and E10-/- mice preferred the previously rewarded location $(\mathrm{t}(38)=2.09$, $\mathrm{p}<0.05$ i.e. above chance level 12.5\%; Figure 4D), i.e. they still preferred the location where reward had been provided during the reversal learning. To assess cognitive function, E10+/- and E10-/- mice were also exposed to a passive avoidance test with a memory probe trial [16]. At baseline, before being exposed to an aversive stimulus, neither of the gene-manipulated mice preferred any of the four corners (Figure 4E; left). During training, the mice avoided the corner in which 

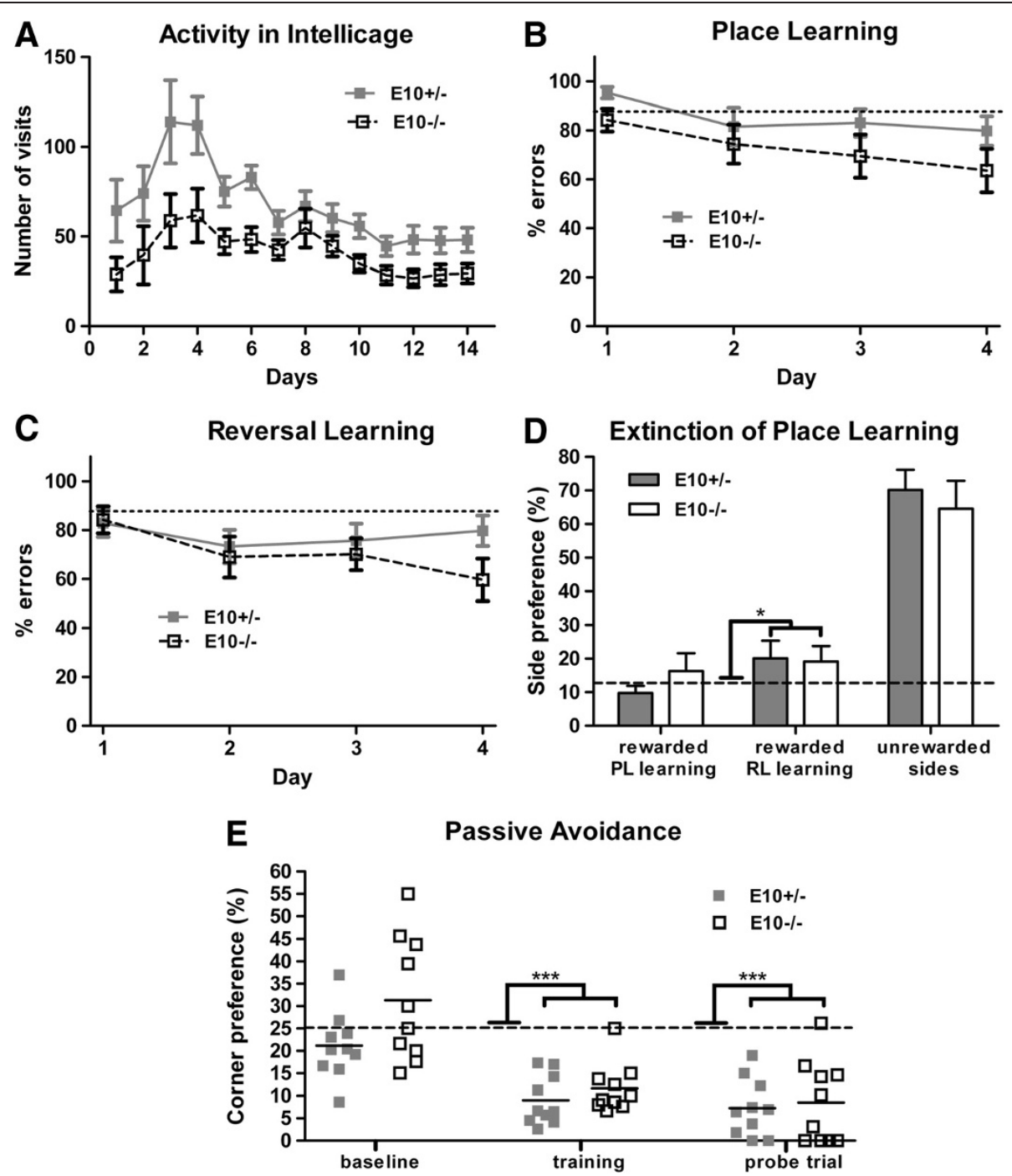

Figure 4 Activity and learning ability of E10+/- and E10-/- mice in IntelliCage. (A) 9-10 months-old mice devoid of tau exon 10 (E10-/-) were less active when habituating to IntelliCages as compared to E10+/- mice (E10+/-, $n=10 ; E 10-/-; n=12)$. No genotype-dependent differences were observed during (B) Place learning, (C) Reversal learning and (D) Extinction of place learning (E10+/-, $n=10 ; E 10-/-; n=10)$. (D) The results were indicative of response perseverance since the previously rewarded corner (rewarded RL learning; $p<0.001$ ) was preferred when compared to chance level (dotted line, 12.5\%) when all locations were again made accessible for drinking. (E) In passive avoidance, the mice significantly avoided those corners in which an aversive stimulus had been given during training $(p<0.001)$ and probe trial $(p<0.001)$ when compared to chance level (dotted line, $25 \%$ ), i.e. the animals learnt and remembered but there were no genotype-dependent differences in associative learning or memory. ${ }^{*} p<0.05 ;{ }^{* *} p<0.01 ;{ }^{* * *} p<0.001$.

they were exposed to an aversive stimuli (an air-puff), but there was no genotype-dependent difference (training $\mathrm{t}(18)=1.07, \mathrm{p}=0.30$; probe trial $\mathrm{t}(18)=0.35, \mathrm{p}=0.73$; chance level is $25 \%$; Figure $4 \mathrm{E}$; middle). In the probe trial, both E10+/- and E10-/- mice avoided the corner where they had previously been exposed to an aversive stimuli (training $\mathrm{t}(38)=11.9, \mathrm{p}<0.001$; probe trial $\mathrm{t}(38)=9.84$, $\mathrm{p}<0.001$; chance level is $25 \%$; Figure 4E; right). These findings were indicative of learning and memory retention abilities in both groups of gene-manipulated mice.
No genotype-dependent effects on anxiety-like or exploratory behaviours

Since, E10-/- mice were less active than E10+/- mice in the intelliCages we wanted to further examine locomotion, emotionality and explorative behaviours with traditional behavioural tests. Behaviour in elevated plus maze is thought to reflect anxiety and risk aversion, while open field measures activity and willingness to explore novel environment. There were no genotype-dependent differences in the open field test. The different mice did not differ in terms of 


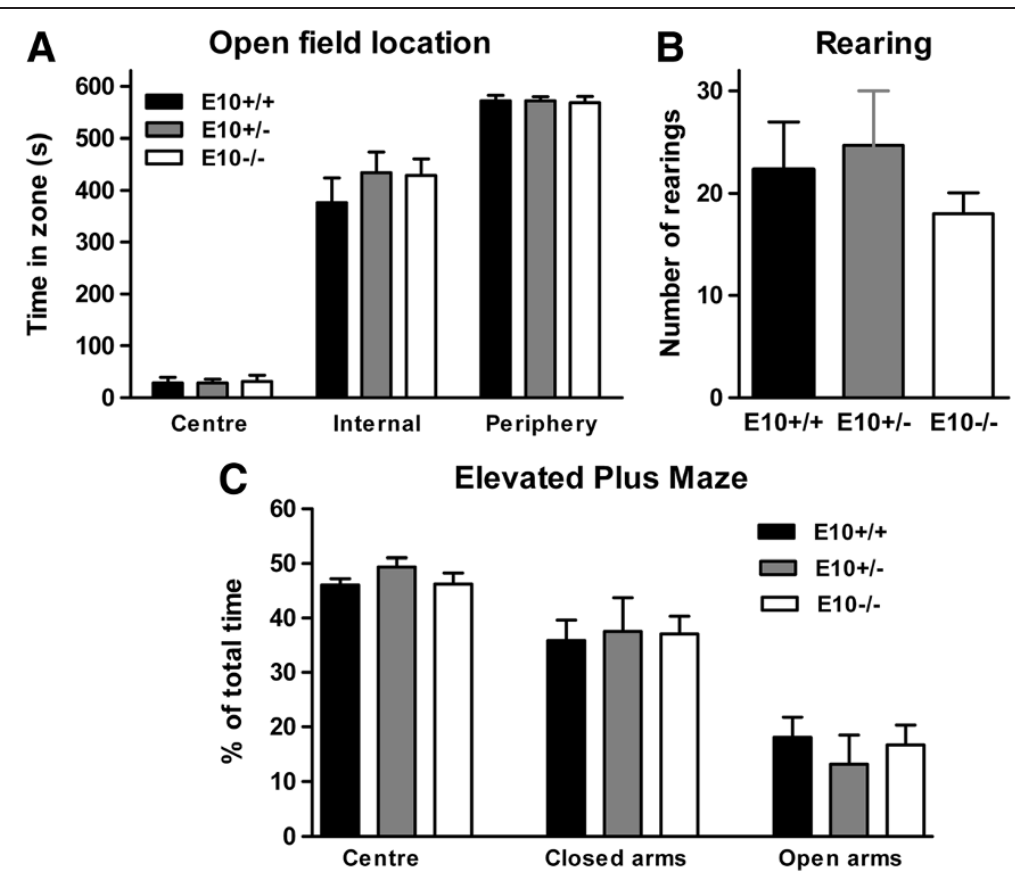

Figure 5 Exploratory behaviour of wild-type, E10+/- and E10-/- mice. (A) 12-16 months-old wild-type (E10+/ $+; n=9), E 10+/-(n=7)$ and E10-/- $(n=6)$ mice explored the different compartments of the open field apparatus to the same extent. (B) Rearing activity was similar between the genotypes. (C) Behaviour in the elevated plus maze did not depend upon partial or complete ablation of exon 10.

location preference (center $\mathrm{F}(2)=0.03, \mathrm{p}=0.97$ ); internal $\mathrm{F}(2)=0.04, \mathrm{p}=0.96)$; periphery $\mathrm{F}(2)=0.60, \mathrm{p}=0.56$; Figure 5A), distance moved (Additional file 1: Figure S3) or rearing activity $(F(2)=0.49, p=0.62$; Figure $5 B)$. Similarly in the elevated plus maze, gene-manipulated mice were equally unwilling to spend time in the open arms as wild-type $(\mathrm{E} 10+/+)$ mice (center $\mathrm{F}(2)=1.39, \mathrm{p}=0.27)$; closed arms $\mathrm{F}(2)=0.038, \mathrm{p}=0.96$ ); open arms $\mathrm{F}(2)=0.36$, $\mathrm{p}=0.70$; Figure 5C).

\section{Deletion of exon 10 does not result in gross morphological abnormalities or tauopathy}

Brains of 12 months-old wild-type (E10+/+), E10+/- and E10-/- mice were examined for major structural changes. Sections were stained with hematoxylin and luxol fast blue dyes, but we did not find any genotype-dependent differences in the forebrain, cerebellum or spinal cord (Figure 6). The astroglial marker, glial fibrillary acidicprotein (GFAP) was used as a rather unspecific sensor of abnormalities in brain tissue. However, we did not find any differences between the mice (Figure 7). Similarly, there were no genotype-dependent differences in GFAP-staining in cerebellar tissue (data not shown) and no macroscopic differences of whole brain (Additional file 1: Figure S4). We were unable to find any evidence of tau aggregation in the brains of E10+/- or E10-/- mice (data not shown).

\section{Discussion}

The cytoskeleton helps cells maintain a dynamic cellular morphology in response to the demands of the surrounding environment. Microtubule-binding proteins likely play a major role in cytoskeletal integrity which is highly critical to neuronal functions and viability, since it enables bidirectional axonal transport of vesicles and organelles along extended processes. In the adult human brain, splicing of tau protein gives rise to a 1:1 balanced expression of $3 R$ - and $4 R$-tau mRNA, while in rodents only $4 R$-tau mRNA is expressed in adult animals. Interestingly, in mouse brain exon 10 is not utilized for protein synthesis until postnatal stages $[11,17]$, when the genome might be less able to activate compensatory mechanisms and mask phenotypes. This is the first study in which the splicing pattern of tau, a microtubule-binding protein, has been altered in the genome with the purpose of studying physiological functions of exon 10 in vivo. Gene targeting is typically associated with the creation of knockout mice, often generated by ablating the basal promoter and exon 1 . There are a few reports in which the technique has been used to solely eliminate a single exon [18]. In the current study, equal amounts of 3R- and 4R-tau protein were synthesized in 2 months-old E10+/- mice while wild-type littermates only expressed 4R-tau protein. Moreover, immunostaining with a 3R-tau selective antibody confirmed axonal localization of tau in genetically 


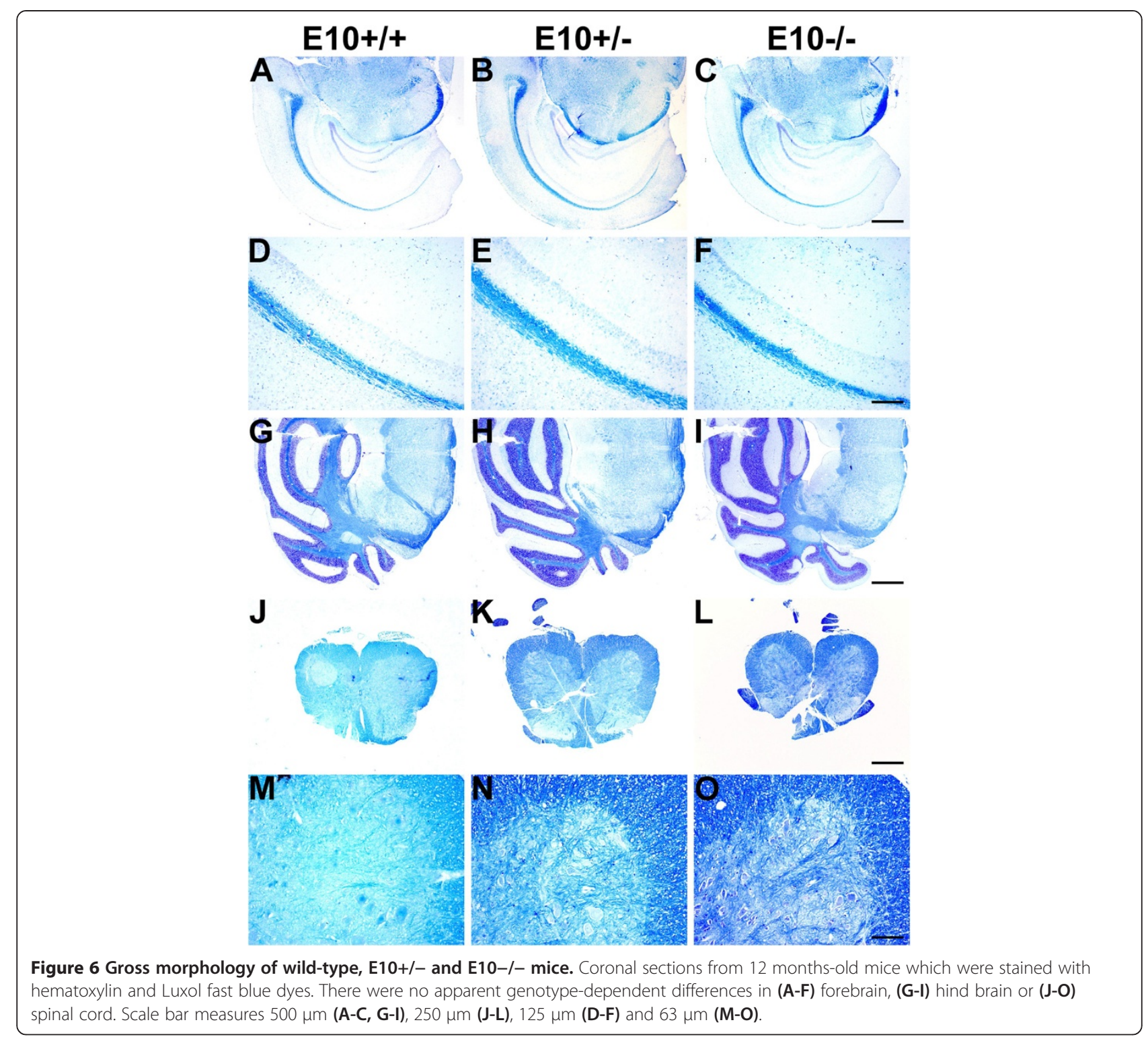

manipulated mice (E10+/-). Altogether, gene targeting technique led us to the successful generation of genemanipulated mice expressing 3R:4R-tau (E10+/-) and $3 R$-tau (E10-/-) mRNA and protein with expected subcellular localization.

Functional studies revealed an age-dependent decline of sensorimotor skills only in middle-aged E10-/- mice. Adult and middle-aged E10+/- mice had similar motor skills in the rotarod test as E10+/+ mice, consistent with haploinsufficiency seldom giving phenotypes in knockout studies. Interestingly, adult (5-6 months) E10+/- mice were actually stronger than E10+/+ mice, which could be explained by activation of compensatory mechanisms. It has been suggested that other microtubule-associated proteins, MAP1A and MAP1B, are involved in functional compensation. Ablation of tau resulted in $\sim 2$-fold increased MAP1A levels in young mice ( 2 weeks old) $[2,4]$, while levels of MAP1B and other MAPs remained unchanged. Compensatory mechanisms seem more active during embryogenesis and early postnatal development, since MAP1A was unchanged in 12 months-old tau knockout mice [4]. We speculate that functional compensation in adult E10-/- mice was overridden by the effects of aging, resulting in sensorimotor deficits.

Cognitive and emotional functions in the genemanipulated mice were examined, partly because tau anomalies are related to dementia disorders. We decided to restrict IntelliCage analyses to two groups of mice; E10+/ - and E10-/ - because we wanted to attain a reasonable statistical power. The decision was also due to 


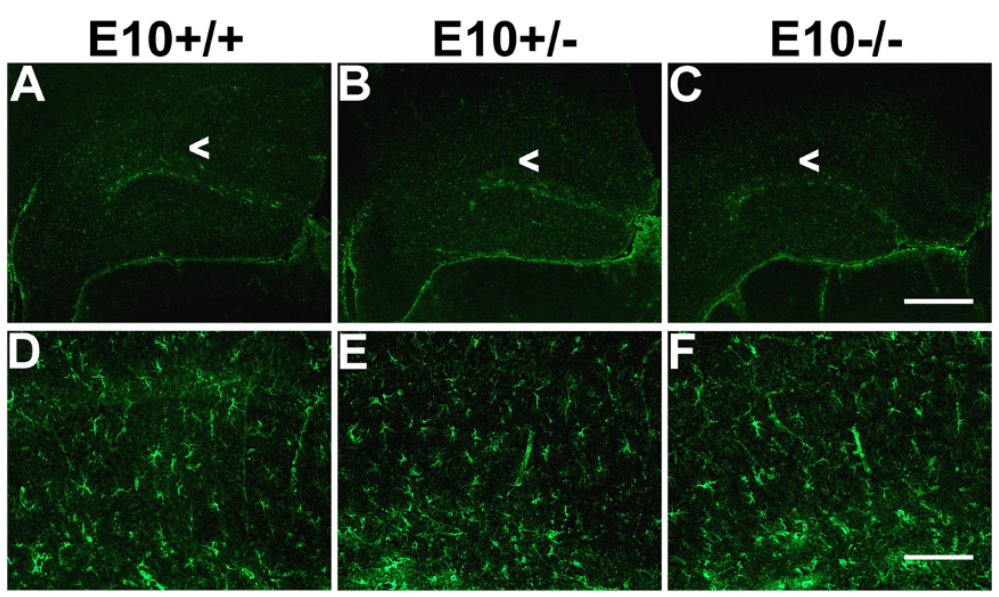

Figure 7 Astroglial immunostaining in the hippocampus of wild-type, E10+/- and E10-/- mice. Coronal sections from 12 months-old mice were stained with a GFAP-antibody. Differences in glial staining were not apparent in E10+/- and E10-/- mice when compared to wildtype (E10+/+) mice. Magnification of CA1 region is marked with arrowheads. Scale bars measure (A-C) $500 \mu \mathrm{m}$ or (D-F) $125 \mu \mathrm{m}$.

availability of IntelliCages and female mice well matched for age. Moreover E10+/- and E10+/+ mice had behaved essentially the same in the sensorimotor tests. These observations were consistent with many other knockout studies, in which haploinsufficiency seldom results in phenotypes. E10-/- mice were less active than E10+/- mice in the IntelliCages, observations which were not reflected in differential anxiety-like behaviours or diminished exploratory behaviour when animals were tested in open field or elevated plus maze. In IntelliCages, mice are housed for days undisturbed in social groups in an environment to which they are allowed to habituate. The procedures of open field and elevated maze testing are markedly different, and this can of course have an impact on the outcome. Both groups habituated well to the new environment and did not show overt cognitive dysfunctions in IntelliCages. We conclude that deletion of exon 10 in tau does not result in cognitive or emotional phenotypes, although it remains to be investigated if this occurs with aging.

Taken together phenotypes in mice genetically manipulated around exon 10 in tau were limited to sensorimotor defects and partly similar to those found in tau knockout mice $[3,19]$. Impaired performance might relate to cerebellar dysfunctions, since this brain region to a large extent develops at postnatal stages when splicing of tau-mRNA is abnormal in E10+/- and E10-/- mice [20]. We were unable to find gross morphological changes, but there could be subtle differences in neural tree or synaptodendritic connections leading to network dysfunctions. Electrophysiological and high-resolution quantitative morphology would be needed to investigate such changes, studies which we consider to be outside the scope of an initial characterization of the models.
There is evidence to suggest that in tauopathies, the pathological aggregation of tau can be primary pathogenic e.g. in frontotemporal lobe dementia (FTLD-17) or secondary pathogenic e.g. to $\mathrm{A} \beta$-aggregation in $\mathrm{AD}$ [21]. However, it remains unclear why human $A \beta$ accumulation by itself does not generate neurofibrillary tangles in A $\beta P P$ transgenic mice. Perhaps structural differences make murine tau unable to form fibrils but in vitro experiments contradict this theory [22]. Murine tau can take part in tau aggregation in vivo but so far seems unable to initiate tau pathology by itself [23-25]. An unknown factor or a trigger, such as misfolded or aggregated human tau or perhaps $A \beta$ seems to be critical. The differences in splicing of tau in mouse and human brain, i.e. the 1:1 balanced 3R-/4R-tau mRNA splicing pattern, could directly impact on the stability of the microtubule structure and on aggregation of tau. It remains to be investigated whether the microtubule system of E10+/- and E10-/- mice is more vulnerable to a trigger of tau-misfolding e.g. an aggregate of human $A \beta$ or human tau than wild-type mice harbouring intact murine tau $(\mathrm{E} 10+/+)$.

\section{Conclusions}

This is the first functional study of exon 10 in tau, which is linked to neurodegenerative disease. By deleting exon 10 it was possible to alter alternative splicing of tau such that 3R-tau protein was synthesized instead of $4 \mathrm{R}$-tau protein. The anatomic and subcellular localization of tau protein synthesis was maintained in the genemanipulated animals. Middle-aged mice lacking exon 10 in tau (E10-/-), expressed only 3R-tau protein and displayed mild sensorimotor deficits with reduced grip strength and motor coordination. Haploinsufficient mice 
(E10+/-), expressing both 3R- and 4R-tau protein, behaved nearly always equal to wild-type mice $(\mathrm{E} 10+/+)$ which expressed only $4 \mathrm{R}$-tau protein. There were no genotypedependent effects on cognition, emotionality or on gross brain structure and tissue morphology. Thus, sensorimotor functions depend on tau exon 10, while perhaps additional functions are masked by related microtubulebinding proteins.

\section{Methods}

\section{Ethical note}

Appropriate experimental procedures were approved by the committee of ethical conduct in research on animal at the Uppsala University (ethical permits C17/7 (200703-02 - 2010-03-02), C374/9 (2010-01-29 - 2013-01-29) and C278/12 (2012-12-20 - present) and performed according to guidelines of ethical conduct on science in compliance with local animal care.

\section{Gene targeting}

A 2.2-kbp genomic fragment in intron 9 of the murine tau gene was amplified with genomic DNA from R1 embryonic stem (ES) cells as template using high-fidelity Expand PCR system (Roche, Basel, Switzerland). Primers started at 15718139 and 15720351 respectively in NT_165773.2. Likewise a 3.6-kbp fragment stretching from intron 10 into intron 11 was amplified with primers starting at 15720889 and 15724540 in NT_165773.2. Primers were designed as to avoid $\sim 200$ bp intron sequences located in introns 9 and 10 , immediately adjacent to exon 10 . Both genomic fragments were inserted into the pBluescript II KSvector where they flanked a $2 \mathrm{kbp}$ fragment containing a neomycin gene surrounded by loxP-sites that had been excised from the pKK7-vector. The targeting construct was checked by multiple restriction enzyme digests and fully sequenced. Only a few mismatches were found as compared to the NCBI database. None of those were located in or adjacent to protein coding sequences. The targeting construct was introduced into R1 ES cells by electroporation and homologous recombination was verified in 7 out of 375 individual clones by screening with two long-range PCR reactions. In both reactions, one primer annealed to sequences outside the targeted allele (starting at 15717864 and 15724785 respectively) while the other primer annealed to sequences within the neomycin (neo) gene. Male chimeras were obtained by microinjection of ES cell clones into blastocysts and subsequent transplantation of these blastocysts to CD1 pseudopregnant mice. The blastocysts were generated by mating with $\mathrm{C} 57 \mathrm{BL} / 6 \mathrm{NCrl}$ females and B6D2F1/Crl males, Male chimeras were bred with female $\mathrm{C} 57 \mathrm{Bl} /$ 6JBomTac mice (Taconic, Hudson, NY, USA) as to generate female offspring, which were then bred with male transgenic mice expressing phosphoglycerate kinase
(pgk)-CRE recombinase (BALB/c × C57B1/B6)F1 [26]. The loxP-flanked neo-cassette was thereby deleted from the targeted allele in some of the offspring.

\section{PCR analyses of genomic DNA and mRNA}

Genomic DNA from ear-biopsies was prepared as previously described [27]. PCR amplification was done with primers annealing at 15720175 and 15721073 in NT_165773.2 as to frame exon 10 in the murine tau gene and to generate $\sim 900$ or $\sim 460$ bp bands depending upon the inclusion or exclusion of exon 10. Alternatively the targeted allele was identified by using with one primer located in the $\sim 100$ bp sequence adjacent to the remaining loxP-site, and therefore unique to the targeted allele, and the other primer in nearby intron sequences of the murine tau gene. Total RNA was purified with RNA STAT-60 (Tel-Test, Friendwood, TX, USA), which is based on the guanidine thiocyanate method. Universal Riboclone cDNA synthesis system (Promega, Madison, WI, USA) and an antisense primer located at 1281-1262 in NM_010838.3 were used for first strand synthesis. The nascent cDNA was then PCR amplified with primers which annealed at 671-690 (in exon 9) and 975-954 (in exon 11) in NM_010838.3 as to simultaneously assay 3R-tau and $4 R$-tau mRNA expression. Alternatively cDNA was amplified with primers located at 671-690 (in exon 9) and 893876 (in exon 10) whereby only $4 R$-tau mRNA was detected. The PCR products were separated by electrophoresis on ethidium bromide-stained agarose gels, and examined and photographed under UV-light.

\section{Histology and western blot}

Mice were anesthetized with $0.4 \mathrm{ml}$ Avertin $(25 \mathrm{mg} / \mathrm{ml})$ and intracardially perfused with $0.9 \%(\mathrm{w} / \mathrm{v})$ saline solution. Brains were dissected and either directly frozen on dry ice for western blot analyses, or instantly frozen in 2-methylbutane at $-30^{\circ} \mathrm{C}$ with dry ice and used for immunohistochemistry. All tissues were stored at $-80^{\circ} \mathrm{C}$ until use. Sections, $14 \mu \mathrm{m}$, were cut in a cryostat, fixed in $4 \%$ formalin for $5 \mathrm{~min}$, permeabilized in $0.4 \%$ Triton $\mathrm{X}-100$ for $5 \mathrm{~min}$ and treated with $0.3 \% \mathrm{H}_{2} \mathrm{O}_{2}$ for $15 \mathrm{~min}$ to eliminate endogenous peroxidase activity. Tissues were then incubated with DAKO-block for $1 \mathrm{~h}$ to block unspecific signal and then with primary antibodies 3RD $(x 40)$ and Tau-1 $(\times 200)$ (Millipore, Billerica, MA, USA) over night at $+4^{\circ} \mathrm{C}$. Sections were then washed and treated with secondary antibodies, $\mathrm{ABC}$-reagents and developed with NOVA RED as described [28]. For Cresyl violet and Luxol fast-blue staining, mice were intracardially perfused with $4 \%(\mathrm{w} / \mathrm{v})$ formaldehyde solution and neuronal tissues were dissected and immersed in this solution for 3 days, dehydrated in etOH ( $40 \% 2 \times 4$ h; $70 \%$ o.n.; $80 \% 2 \times 1.5$ h; $95 \% 2 \times 1.5 \mathrm{~h} ; 100 \% 2 \times 1 \mathrm{~h}$; Xylen $2 \times 30 \mathrm{~min}$ ), immersed in paraffin o.n at $62^{\circ} \mathrm{C}$, embedded and sectioned at $5 \mu \mathrm{m}$. 
Brains used for western blot were extracted in $10 \mathrm{mM}$ Tris, $0.8 \mathrm{M} \mathrm{NaCl}, 1 \mathrm{mM}$ EDTA, 10\% (w/v) sucrose supplemented with Complete ${ }^{\bullet}$ (1:50) protease inhibitor cocktail (Roche, Basel, Switzerland) and centrifuged at $26000 \times g$ for $20 \mathrm{~min}$ at $+4^{\circ} \mathrm{C}$. The pellet was extracted and centrifuged again at $26000 \times g$ for $20 \mathrm{~min}$ at $+4^{\circ} \mathrm{C}$. The supernatants were pooled and supplemented with $1 \%(\mathrm{w} / \mathrm{v})$ sarkosyl and incubated by rotary shaking at 200 RPM for $1 \mathrm{~h}$ at RT. The mixture was then centrifuged at 100 $000 \times g$ for $1 \mathrm{~h}$ at $+4^{\circ} \mathrm{C}$ and the sarkosyl-soluble supernatants were run on $8 \%$ Tris-Glycine gels (InVitrogen, Carlsbad, CA, USA) and analyzed with western blot as previously described [28]. A human recombinant tau protein ladder was used as size markers (rPeptide, Bogart, GA, USA). Nitrocellulose filters were incubated with primary antibodies 3RD (x20 000) and Tau-1 (x50 000) in 5\% (w/v) non-fat dry milk in $1 \times$ TTBS for $1 \mathrm{~h}$ at RT.

\section{Rotarod}

Motor coordination of wild-type $(\mathrm{E} 10+/+), \mathrm{E} 10+/-$ and E10-/- mice was assessed at 5-7 months and 13-17 months of age. The ROTA-ROD apparatus (dimensions $362 \times$ $240 \times 400 \mathrm{~mm}$ ) (model LE8200, Panlab, S.L.U., Spain) consisted of a vertical plastic (methacrylate) rotating rod (dimensions $50 \times 30 \mathrm{~mm}$ ) with a ribbed surface flanked by two large discs (arnite). Each mouse was given three trials with $10 \mathrm{~min}$ inter-trial interval for three consecutive days. Trials started at a minimum speed of $4 \mathrm{rpm}$ followed by 5 min acceleration time (maximum speed $40 \mathrm{rpm}$ ) with increase of speed with $1 \mathrm{rpm}$ every $8^{\text {th }}$ second. The latency to fall and the rotation speed when the mice fell was recorded. The apparatus was cleaned with $70 \%$ ethanol between trials.

\section{Grip strength}

Muscular strength of wild-type $(\mathrm{E} 10+/+), \mathrm{E} 10+/-$ and E10-/ - mice was evaluated at 5-7 months and 1317 months of age. The grip strength meter (model GS3, BIOSEB, Chaville, France) consisted of a stainless steel grid (dimensions $100 \times 80 \mathrm{~mm}$ ) with a sensor capacity ranging 0-20 Newton (N). Each mouse was held by the tail, placed on the grid with all four paws and gently pulled horizontally by the tail along a straight line until the mice released the grid and the maximum forced was recorded. Each mouse was given three trials with 30 seconds intertrial interval for four consecutive days. The stainless grid was cleaned with $70 \%$ ethanol between trials.

\section{IntelliCage}

Behaviours of female E10+/- and E10-/- mice housed in social groups were tested at 9-11 months of age. The protocols used have been previously described in detail [14]. Each IntelliCage was equipped with four learning corners accessible through a ring antenna which led to
Table 1 Behavioural analyses in the IntelliCage

\begin{tabular}{ll}
\hline Name of the module & Duration (days) \\
\hline 1. Free exploration (FE) & 1 \\
2. Habituation & 14 \\
3. Place learning (PL) & 4 \\
4. Reversal place learning (RevPL) & 4 \\
5. Extinction of place preference & 2 \\
6. Passive avoidance & 3 \\
\hline
\end{tabular}

A) Place learning (PL): In a designated corner for each mouse, a nosepoke opened the left door once during a visit. Thereby the animals were given access to $300 \mathrm{mM}$ sucrose (rewarded corner) during two drinking sessions per night (23:00-24:00 and 04:00-05:00). The right door in the same corner remained closed. In the other three corners, a nosepoke opened the right door which gave access to water (unrewarded corner) once during the two drinking sessions. The left door remained closed during the rest of time. The corner in which reward was given was randomized and individual designated to each mouse.

(B) Reversal learning during drinking sessions (revPL): The same protocol as PL except that the respective corner giving access to sucrose was the diagonal opposite.

two mechanical doors controlling access to two water bottles. Light-emitting diodes (LEDs; colour code 1638) were located above each door inside the learning corners. Three events were recorded in the learning corners; visit, nosepoke and lick. A visit was recorded when the ring antenna detected the microchip and the heat sensor simultaneously sensed a temperature shift. A nosepoke was recorded when the infrared beam in front of the mechanical door was interrupted. A lick was recorded when a mouse touched the nipple of the water bottle such that the "lick sensor" exceeded a threshold level. IntelliCage software (ver. 2.4.2) and IntelliCage corners (ver. 2.0) were used (http://www.newbehavior.com). The animals were allowed to habituate to the testing room for a week, and were then under isoflurane anaesthesia subcutaneously injected with transponders in the interscapular area. At least $24 \mathrm{~h}$ after transponder implantation, the mice were placed into the IntelliCages at the beginning of the dark phase. The duration of IntelliCage housing was 6 weeks, during which the dark phase was scheduled from 20:00 until 08:00 and light phase 08:00-20:00. The modules, which are listed in Table 1, have previously been extensively reported [14], and here only deviations from those protocols are described:

\section{Elevated plus maze}

Anxiety-like behaviours of 12-16 months-old wild-type $(\mathrm{E} 10+/+), \mathrm{E} 10+/-$ and E10-/- mice were assessed in a maze made of grey coloured PVC walls (model LE842, Panlab S.L.U., Barcelona, Spain). It consisted of two open arms and two closed arms (length $29.5 \mathrm{~cm}$ and width $6 \mathrm{~cm}$ ) arranged as a cross. The center was $6 \times 6 \mathrm{~cm}$ and the closed arms were protected by a $15 \mathrm{~cm}$ wall made of grey PVC. Individual mice were placed at the center of the maze 
facing the open arms for a single $5 \mathrm{~min}$ trial. Their behaviours were recorded on video and analysed with Smart Junior program V1.0. The maze was cleaned with $70 \%$ ethanol between trials.

\section{Open-field test}

A transparent cage (width $37 \mathrm{~cm}$, length $48 \mathrm{~cm}$ and height $20 \mathrm{~cm}$ ) was used to assess locomotor activity, exploratory and anxiety-like behaviours of 12-16 months-old wild-type $(\mathrm{E} 10+/+), \mathrm{E} 10+/-$ and E10-/- mice. Individual mice were placed at the center of the cage for $10 \mathrm{~min}$ and their behaviour was recorded with a video camera. The testing cage was divided in three zones: periphery, internal and center comprising $43.6 \%, 48.6 \%$ and $7.8 \%$ of the total area respectively. Off-line analysis of the records was performed with Smart Junior program V1.0. The area was cleaned with $70 \%$ ethanol between trials.

\section{Statistical analyses}

GraphPad (San Diego, CA, USA) and Statistica (Tulsa, OK, USA) were used. Data which had been verified not to deviate from a normal distribution were analyzed with unpaired $t$-test, one-way or two-way ANOVA, or otherwise with a non-parametric test e.g. Mann-Whitney U-test. Outliners were identified with Grubbs' test, and post-hoc test of one-way and two-way ANOVA were analyzed with Fisher LSD. Results presented are mean \pm s.e.m. unless otherwise stated.

\section{Additional file}

Additional file 1: Figure S1. A graphical illustration of the genomic structure around exon 10 on both alleles in the murine tau gene locus, tau mRNA expression and tau protein synthesis in wild-type (E10+/+, upper), E10+/- (middle) and E10-/- mice (lower). A part of the tau locus with exons 9, 11 (yellow), 10 (orange, dotted lines) and genetic deletion $(\mathbf{)})$ is shown. The four microtubule domains (R1-R4) in tau protein are encoded by exons 9-12 in tau. Alternative splicing of tau in adult wild-type mouse brain results in 4R-tau protein being synthesized by both alleles (upper). Ablation of exon 10 on one of the two alleles in tau (E10+/- mice) should theoretically lead to a 1:1 balanced ratio of 3R- and 4R-tau protein synthesis (middle). This mixture of 3R-/4R-tau protein synthesis is found in adult human brain. In contrast, lack of exon 10 on both alleles in tau (E10-/-) should results in 3R-tau protein synthesis by both alleles (lower). Figure $\mathbf{S 2}$. Sensorimotor functions of wild-type (E10+/+), E10+/- and E10-/- mice. 13-17 months-old mice devoid of tau exon 10 (E10-/-) were also impaired in rotarod when maximum speed was recorded compared to E10+/+ $(p<0.05)$ and E10+/- mice $(p<0.01)$ $(E 10+/+, n=18 ; E 10+/-, n=16 ; E 10-/-; n=15) .{ }^{*} p<0.05$ and ${ }^{* *} p<0.01$. Figure S3. Exploratory behaviours of wild-type E10(+/+), E10+/- and E10-/- mice. At 12-16 months of age, E10+/- and E10-/- mice travelled a similar distance in the open field apparatus as wild-type mice. Figure S4. No macroscopic differences between brains of wild-type (E10+/+) and $\mathrm{E} 10+/$ - and $\mathrm{E} 10-/$ - mice.

\section{Abbreviations}

AßPP: Amyloid- $\beta$ precursor protein; A $\beta$ : Amyloid- $\beta$; CA1: Cornu ammonis 1; CRE: Causes recombination; E10: Exon 10; ES: Embryonic stem; FE: Free exploration; FTLD-17: Frontotemporal lobe dementia (FTLD-17); GFAP: Glial fibrillary acidic-protein; LEDs: Light emitting diodes; MAPs: Microtubule-associated proteins; NCBI: National center for biotechnology information; RT-PCR: Reverse transcriptase polymerase chain reaction; 3R: 3 repeat; pgk: Phosphoglycerate kinase; P1: Postnatal day 1; PL: Place learning; PVC: Polyvinyl chloride.

\section{Competing interests}

In this study there are no actual or potential conflicts of interests.

\section{Authors' contributions}

LN conceived the study, designed and generated the gene targeting vector and screened embryonic stem cell clones. Uppsala University Transgenic Facility cultured embryonic stem cells and performed blastocyst injection and implantation. AG was responsible for animal care and breeding. She planned and carried out behavioural studies and associated data analyses; LN and AG carried out immunohistochemical and biochemical experiments, and together designed experiments and wrote ethical applications. AG did statistical analyses under the supervision of LN. LN, AG and LL wrote the manuscript. All authors read and approved the final manuscript.

\section{Acknowledgements}

This work was funded by grants from Uppsala University, Landstinget i Uppsala län, the Swedish Research Council [\#2009-4567) LL, (\#2009-4389) LN], The Swedish Brain Foundation, Alzheimerfonden, Gamla Tjänarinnor, Gun och Bertil Stohne (AG, LN), Magnus Bergvall, Åhlénsstiftelsen, Lundströms Minne, Frimurarstiftelsen, Trolle-Wachmeisters stiftelse (LN). Klas Kullander is greatly acknowledged for giving the pKK7-vector and Åsa Mackenzie for providing access to pgk-CRE recombinase transgenic mice. The Uppsala University Transgenic Facility (UUTF) is greatly acknowledged for their support in developing the E10+/- and E10-/- models. Micaela Vrede is acknowledged for help with functional studies, Erik Sundström and Lena Holmberg for dissecting spinal cord, and Kristina Magnusson for photography.

Received: 21 August 2013 Accepted: 19 November 2013 Published: 22 November 2013

\section{References}

1. Dehmelt $L$, Halpain $S$ : The MAP2/Tau family of microtubule-associated proteins. Genome Biol 2005, 6(1):204.

2. Harada A, Oguchi K, Okabe S, Kuno J, Terada S, Ohshima T, Sato-Yoshitake R, Takei Y, Noda T, Hirokawa N: Altered microtubule organization in small-calibre axons of mice lacking tau protein. Nature 1994, 369(6480):488-491.

3. Ikegami S, Harada A, Hirokawa N: Muscle weakness, hyperactivity, and impairment in fear conditioning in tau-deficient mice. Neurosci Lett 2000 279(3):129-132.

4. Dawson HN, Ferreira A, Eyster MV, Ghoshal N, Binder LI, Vitek MP: Inhibition of neuronal maturation in primary hippocampal neurons from tau deficient mice. J Cell Sci 2001, 114(Pt 6):1179-1187.

5. Takei Y, Teng J, Harada A, Hirokawa N: Defects in axonal elongation and neuronal migration in mice with disrupted tau and map1b genes. J Cell Biol 2000, 150(5):989-1000.

6. Andreadis A: Tau gene alternative splicing: expression patterns, regulation and modulation of function in normal brain and neurodegenerative diseases. Biochim Biophys Acta 2005, 1739(2-3):91-103.

7. Janke C, Beck M, Stahl T, Holzer M, Brauer K, Bigl V, Arendt T: Phylogenetic diversity of the expression of the microtubule-associated protein tau: implications for neurodegenerative disorders. Brain Res Mol Brain Res 1999, 68(1-2):119-128.

8. Hutton M, Lendon CL, Rizzu P, Baker M, Froelich S, Houlden H, Pickering-Brown S, Chakraverty S, Isaacs A, Grover A, et al: Association of missense and 5 '-splice-site mutations in tau with the inherited dementia FTDP-17. Nature 1998, 393(6686):702-705.

9. Hong M, Zhukareva V, Vogelsberg-Ragaglia V, Wszolek Z, Reed L, Miller BI, Geschwind DH, Bird TD, McKeel D, Goate A, et al: Mutation-specific functional impairments in distinct tau isoforms of hereditary FTDP-17. Science 1998, 282(5395):1914-1917.

10. Kosik KS, Orecchio LD, Bakalis S, Neve RL: Developmentally regulated expression of specific tau sequences. Neuron 1989, 2(4):1389-1397.

11. Takuma $\mathrm{H}$, Arawaka $\mathrm{S}$, Mori H: Isoforms changes of tau protein during development in various species. Brain Res Dev Brain Res 2003, 142(2):121-127.

12. Feinstein SC, Wilson L: Inability of tau to properly regulate neuronal microtubule dynamics: a loss-of-function mechanism by which tau might mediate neuronal cell death. Biochim Biophys Acta 2005, 1739(2-3):268-279. 
13. Binder LI, Frankfurter A, Rebhun LI: The distribution of tau in the mammalian central nervous system. J Cell Biol 1985, 101(4):1371-1378.

14. Codita A, Gumucio A, Lannfelt L, Gellerfors P, Winblad B, Mohammed AH, Nilsson LN: Impaired behavior of female tg-ArcSwe APP mice in the IntelliCage: a longitudinal study. Behav Brain Res 2010, 215(1):83-94.

15. Galsworthy MJ, Amrein I, Kuptsov PA, Poletaeva II, Zinn P, Rau A, Vyssotski A Lipp HP: A comparison of wild-caught wood mice and bank voles in the IntelliCage: assessing exploration, daily activity patterns and place learning paradigms. Behav Brain Res 2005, 157(2):211-217.

16. Voikar V, Colacicco G, Gruber O, Vannoni E, Lipp HP, Wolfer DP: Conditioned response suppression in the IntelliCage: assessment of mouse strain differences and effects of hippocampal and striatal lesions on acquisition and retention of memory. Behav Brain Res 2010, 213(2):304-312.

17. McMillan P, Korvatska E, Poorkaj P, Evstafjeva Z, Robinson L, Greenup L, Leverenz J, Schellenberg GD, D'Souza I: Tau isoform regulation is region- and cell-specific in mouse brain. J Comp Neurol 2008, 511(6):788-803.

18. Gimond C, Baudoin C, van der Neut R, Kramer D, Calafat J, Sonnenberg A: Cre-loxP-mediated inactivation of the alpha6A integrin splice variant in vivo: evidence for a specific functional role of alpha6A in lymphocyte migration but not in heart development. J Cell Biol 1998, 143(1):253-266.

19. Roberson ED, Scearce-Levie K, Palop JJ, Yan F, Cheng IH, Wu T, Gerstein H, Yu GQ, Mucke L: Reducing endogenous tau ameliorates amyloid beta-induced deficits in an Alzheimer's disease mouse model. Science 2007, 316(5825):750-754.

20. Kolb B, Tees RC: The Cerebral cortex of the rat. Cambridge, Mass: MIT Press; 1990.

21. Ballatore C, Lee VM, Trojanowski JQ: Tau-mediated neurodegeneration in Alzheimer's disease and related disorders. Nat Rev Neurosci 2007, 8(9):663-672.

22. Kampers T, Pangalos M, Geerts H, Wiech H, Mandelkow E: Assembly of paired helical filaments from mouse tau: implications for the neurofibrillary pathology in transgenic mouse models for Alzheimer's disease. FEBS letters 1999, 451(1):39-44.

23. Mocanu MM, Nissen A, Eckermann K, Khlistunova I, Biernat J, Drexler D, Petrova O, Schonig K, Bujard H, Mandelkow E, et al: The potential for beta-structure in the repeat domain of tau protein determines aggregation, synaptic decay, neuronal loss, and coassembly with endogenous Tau in inducible mouse models of tauopathy. J Neurosci 2008, 28(3):737-748.

24. de Calignon A, Polydoro M, Suarez-Calvet M, William C, Adamowicz DH, Kopeikina KJ, Pitstick R, Sahara N, Ashe KH, Carlson GA, et al: Propagation of tau pathology in a model of early Alzheimer's disease. Neuron 2012, 73(4):685-697.

25. Liu L, Drouet V, Wu JW, Witter MP, Small SA, Clelland C, Duff K: Trans-synaptic spread of tau pathology in vivo. PloS one 2012, 7(2):e31302.

26. Lallemand $Y$, Luria $V$, Haffner-Krausz R, Lonai P: Maternally expressed PGK-Cre transgene as a tool for early and uniform activation of the Cre site-specific recombinase. Transgenic Res 1998, 7(2):105-112.

27. Conner DA: Mouse colony management. Curr Protoc Mol Biol 2002, 57:23.8.1-23.8.11.

28. Lord A, Kalimo H, Eckman C, Zhang XQ, Lannfelt L, Nilsson LN: The Arctic Alzheimer mutation facilitates early intraneuronal Abeta aggregation and senile plaque formation in transgenic mice. Neurobiol Aging 2006, 27(1):67-77.

doi:10.1186/1471-2202-14-148

Cite this article as: Gumucio et al:: Lack of exon 10 in the murine tau gene results in mild sensorimotor defects with aging. BMC Neuroscience 2013 14:148.

\section{Submit your next manuscript to BioMed Central and take full advantage of:}

- Convenient online submission

- Thorough peer review

- No space constraints or color figure charges

- Immediate publication on acceptance

- Inclusion in PubMed, CAS, Scopus and Google Scholar

- Research which is freely available for redistribution 